\title{
Angular distribution of scission neutrons studied with time- dependent Schrödinger equation
}

\author{
Takahiro Wada ${ }^{1, \star}$, Tomomasa Asano ${ }^{1}$, and Nicolae Carjan ${ }^{2}$ \\ ${ }^{1}$ Department of Pure and Applied Physics, Kansai University, 3-3-35 Yamate-cho, Suita, 564-8680, Japan \\ ${ }^{2}$ Flerov Laboratory of Nuclear Reactions, Dubna, Moscow region, Russia
}

\begin{abstract}
We investigate the angular distribution of scission neutrons taking account of the effects of fission fragments. The time evolution of the wave function of the scission neutron is obtained by integrating the time-dependent Schrodinger equation numerically. The effects of the fission fragments are taken into account by means of the optical potentials. The angular distribution is strongly modified by the presence of the fragments. In the case of asymmetric fission, it is found that the heavy fragment has stronger effects. Dependence on the initial distribution and on the properties of fission fragments is discussed. We also discuss on the treatment of the boundary to avoid artificial reflections.
\end{abstract}

\section{Introduction}

At the moment of scission, the neck that has connected the two fission fragments ruptures. This neck rupture can be very quick because the number of nucleons involved in the neck region is relatively small. After the scission, the neck disappears being absorbed by the fragments leaving some nucleons behind in the neck region and these nucleons are observed as particle emission. The number of scission particles carries the information on the scission process. There have been attempts to estimate the number of scission neutrons theoretically $[1,2]$. The results depend on the nuclear shape such as the neck radius before scission. If we extract the reliable number of scission neutrons from experiments, we can get information on the nuclear shape at the time of scission.

In measuring the scission neutrons experimentally, the information on their angular distribution is essential. The emission of scission neutron is normally supposed to be isotropic in the rest frame of the mother nucleus in the lowest order approximation. However, since they are emitted in the close vicinity of the fission fragments, the final angular distribution of the scission neutron is modified by the fragments through scattering and re-absorption. In the previous study, we proposed a framework to calculate the angular distribution of scission neutrons by solving a time-dependent Schrödinger equation [3]. It was shown that the angular distribution of the scission neutron is strongly modified by the presence of the fragments, namely, the attractive nuclear potential enhances the yields around 0 and 180 degrees, while the absorptive potential diminishes them. We also investigated the angular distribution of scission protons and discussed the effect of the Coulomb field of the fragments [4]. It was shown that the angular distribution of the scission proton is also strongly modified by the fragments. The Coulomb field of the fragments tends to focus the yields around 90 degrees, while the

^e-mail: wadataka@kansai-u.ac.jp 
attractive nuclear potential enhances the yields around 0 and 180 degrees. In this paper, we examine the dependence of the angular distribution of scission neutrons on the initial distribution of neutron and on the properties of fission fragments, such as deformation of the fragments. In the next section, the formulation to calculate the angular distribution of the scission particles is revisited. In Sect. 3, results are presented for the case of the fission of ${ }^{236} \mathrm{U}$ for both symmetric and asymmetric divisions. Next, we discuss on the treatment of the boundary to avoid artificial reflection. Finally, a summary is given.

\section{Framework}

We start with a time-dependent Schrödinger equation (TDSE),

$$
i \frac{\partial \psi}{\partial t}=H \psi, \quad H=-\frac{1}{2 m} \nabla^{2}+U, \quad \hbar=1,
$$

where $\psi$ denotes the neutron wave function, $H$ is the Hamiltonian, and $U$ is the potential that represents the effect of the fission fragments. Assuming the axial symmetry, we solve the TDSE in two-dimensional grid space $(\rho, z)$. The time development is obtained with the use of the mid-point integration,

$$
\psi(t+\Delta t)=\psi(t)-i \Delta t H \psi(t+\Delta t / 2)
$$

By decomposing $\psi$ into the real and the imaginary part, $\psi=P+i Q$, the numerical solution is obtained using the leap-frog method [3]. The potential $U$ has two centers and is parameterized in Woods-Saxon form centered at the position of each fragment. For the case of spherical fragments, $U$ is expressed as,

$$
U(\rho, z)=\frac{V_{0}+i W_{0}}{1+\exp \left(\frac{\sqrt{\rho^{2}+\left(z+B_{2}\right)^{2}}-R_{\mathrm{F} 2}}{a}\right)}+\frac{V_{0}+i W_{0}}{1+\exp \left(\frac{\sqrt{\rho^{2}+\left(z-B_{1}\right)^{2}}-R_{\mathrm{F} 1}}{a}\right)},
$$

where $B_{i}$ is the distance between the center of scission neutron distribution and the $i$-th fragment, $a$ is the diffuseness, and $\mathrm{R}_{\mathrm{F} i}$ is the radius of the potential of $i$-th fragment. The distance $B i$ is given as $B_{i}=\eta \mathrm{R}_{\mathrm{F} i}$, where $\eta$ is the elongation parameter which is to be deduced from the systematics of the total kinetic energy.

We adopt a Gaussian wave packet for the initial wave function of the scission neutron, $\psi(t=$ $\left.0)=C \exp \left(-\left(\alpha_{\rho} \rho^{2}+\alpha_{z} z^{2}\right)\right)\right)$, where $C$ is a normalization constant and $\alpha$ 's denotes the width of the wave packet. We now set a sphere of radius $R$ in the grid space and calculate the neutron flux on this spherical surface,

$$
\mathbf{j}(r, t)=\frac{1}{2 i m}(\psi \nabla \psi *-\psi * \nabla \psi)
$$

We then calculate the number of outgoing scission particles per unit solid angle per unit time and integrate it with time. The angular distribution is given by the number of particles per unit solid angle that passed the surface up to time $t$,

$$
\frac{d N(\theta, t)}{d \Omega}=\int_{0}^{t} \frac{d^{2} N\left(\theta, t^{\prime}\right)}{d \Omega d t} d t^{\prime}=\int_{0}^{t} \mathbf{j}\left(R, \theta, t^{\prime}\right) \cdot \mathbf{n}(R, \theta) R^{2} d t^{\prime}, \quad \mathbf{n}=e_{r}
$$

The energy density and the energy flow are defined respectively as

$$
\varepsilon(\mathbf{r}, t)=\frac{\hbar^{2}}{2 m} \frac{\partial \psi *}{\partial \mathbf{r}} \cdot \frac{\partial \psi}{\partial \mathbf{r}}+U(\mathbf{r}, t) \psi * \psi,
$$


and

$$
\mathbf{j}_{\varepsilon}(\mathbf{r}, t)=\frac{\hbar}{2 i m}\left\{\frac{\hbar^{2}}{2 m}\left(\frac{\partial \psi}{\partial \mathbf{r}} \cdot \nabla \frac{\partial \psi *}{\partial \mathbf{r}}-\frac{\partial \psi *}{\partial \mathbf{r}} \cdot \nabla \frac{\partial \psi}{\partial \mathbf{r}}\right)\right\}+U(\mathbf{r}, t) \mathbf{j}(\mathbf{r}, t) .
$$

The energy that is brought by scission neutrons to a certain angle $\theta$ is calculated in the same manner as in Eq. (5),

$$
\frac{d E(\theta, t)}{d \Omega}=\int_{0}^{t} \mathbf{j}_{\varepsilon}\left(R, \theta, t^{\prime}\right) \cdot \mathbf{n}(R, \theta) R^{2} d t^{\prime}, \quad \mathbf{n}=e_{r},
$$

and the average energy of scission neutron for each direction is given by

$$
\langle\varepsilon(\theta)\rangle=\frac{d E(\theta, t)}{d \Omega} \mid \frac{d N(\theta, t)}{d \Omega} .
$$

\section{Results and discussions}

As an example, we consider the fission of ${ }^{236} \mathrm{U}$ that corresponds to the neutron induced fission of ${ }^{235} \mathrm{U}$. From a systematic study of the average total kinetic energy (TKE) of the fragments, Zhao et al. derived the elongation parameter $h$ which is the ratio between the average distance between the fragments to the contact distance $r_{0}\left(A_{1}^{1 / 3}+A_{2}^{1 / 3}\right)$, where $A_{1}$ and $A_{2}$ are the mass numbers of the fragments and $r_{0}=1.17 \mathrm{fm}$ [5]. The average distance between the fragments is determined so that the corresponding point charge Coulomb energy is equal to the average TKE. They obtained $\eta=1.53$ for the asymmetric fission in $\mathrm{U}$ region. In the calculation, we simply use the same elongation parameter for $B_{i}$ as $B_{i}=\eta r_{0} A_{i}^{1 / 3}$. The distance between the grid points is typically $0.1 \mathrm{fm}$ in both $z$ - and $\rho$ directions. The time step for the integration is typically $\Delta t=0.02 \mathrm{fm} / \mathrm{c}$. The radius $R$ is set to $60 \mathrm{fm}$ and the integration is performed up to $t=5 \times 10^{-21} \mathrm{~s}$. In calculating the time development, we take account of the motion of the fragments due to the Coulomb repulsion between the fragments. In the following, $V_{0}=-40 \mathrm{MeV}$ and $W_{0}=-1 \mathrm{MeV}$ are used for the strength of the potential unless otherwise is mentioned.

\subsection{Dependence on the initial wave function}

First, we investigate the dependence on the initial wave function for the symmetric fission. The initial wave function is assumed to be of the form of $\psi(t=0)=C \exp \left(-\left(\alpha_{\rho} \rho^{2}+\alpha_{z} z^{2}\right)\right)$. The initial average kinetic energy of scission neutron is given as,

$$
\langle\varepsilon\rangle=\frac{\hbar^{2}}{2 m}\left(2 \alpha_{\rho}+\alpha_{z}\right) .
$$

The left panel of Fig. 1 shows the angular distribution of scission neutrons for the isotropic initial wave function $\left(\alpha_{\rho}=\alpha_{z}\right)$ with $\langle\varepsilon\rangle=0.5,1.0,1.5$, and $2.0 \mathrm{MeV}$. The yields along fission axis, 0 and 180 degrees, increase as $\langle\varepsilon\rangle$, while the yield around 90 degrees is almost independent of $\langle\varepsilon\rangle$.

In the right panel of Fig. 1, we show the results for the cases of deformed initial wave function. The value of $\alpha_{\rho}$ is fixed and $\alpha_{z}$ is given as $\alpha_{\rho} / \alpha_{z}=1,9 / 4,4,25 / 4$. When we increase the deformation of the initial wave function, we obtain less yields around 90 degrees, while the yields around 0 and 180 degrees do not change as much. As a whole, the effect of the attractive nuclear potential is to increase the yeilds around 0 and 180 degrees. 

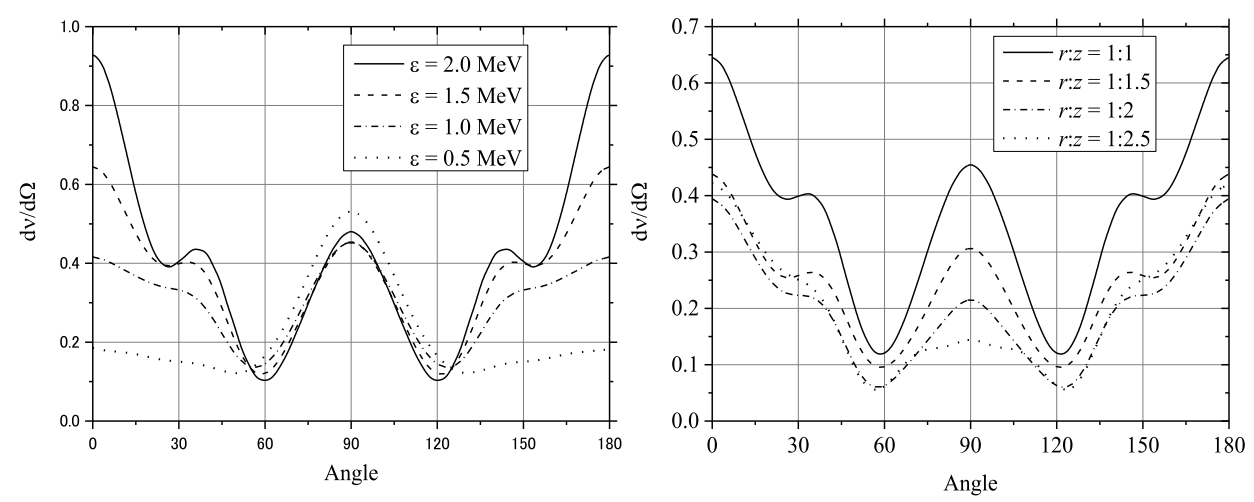

Figure 1. Angular distribution of scission neutron. Left panel: dependence on the width of the wave packet, Right panel: dependence on the deformation of the wave packet.

\subsection{Dependence on the motion and the deformation of the fragments}

Next, we investigate the case of asymmetric fission. The mass number of the heavy fragment is assumed to be $A_{1}=140$. The left panel of Fig. 2 displays the dependence on the motion of the fragments. Three cases are shown; the dashed line shows the result when the fragments are fixed at the initial position, the solid line corresponds to the case that the fragments start from the same position as the first case at rest then accelerated by the Coulomb repulsion, and the dotted-dash line is the case with the pre-scission kinetic energy of $15 \mathrm{MeV}$. In the second and the third case, we keep the TKE unchanged. Note that heavier fragment is on 0 degree and the lighter one is on 180 degrees. As is seen in Fig. 2, contrary to the experimental observation of the angular distribution of prompt neutrons, we observe more neutrons in the direction of heavier fragment. This indicates either that there is a significant contribution of the post scission emission of neutrons from fission fragments or that we need to employ more sophisticated initial condition of scission neutrons [2]. By comparing the solid and the dashed lines, we see that the change of the yield is smaller on the heavier fragment side than that on the light fragment side. The oscillation around 90 degrees is more enhanced for the case of the fixed fragments. That means that the yield around 90 degrees is sensitive to the position of the fragments. This tendency is confirmed when we compare the solid line with the dotted-dash line.

We have assumed spherical fragments so far. Now we examine how the angular distribution changes when the fragments are deformed. We assume that the fragments are deformed axialsymmetrically along the fission axis and the optical potential $U(\rho, z)$ is calculated accordingly. The surface of a fragment is expressed as,

$$
R(\theta)=R_{\delta}(1+\delta \cos \theta) .
$$

where $R_{\delta}$ is determined to conserve the volume of the fragment. We show the results in the right panel of Fig. 2 for several cases. It is seen that the angular distribution on the heavier fragment side is sensitive to the deformation of heavier fragment and vice versa.

\subsection{Angular distribution of the average energy}

Before discussing the average energy of scission neutrons, we will briefly remark on the effects of absorption by the fragments. The left panel of Fig. 3 shows the comparison of the angular distribution 

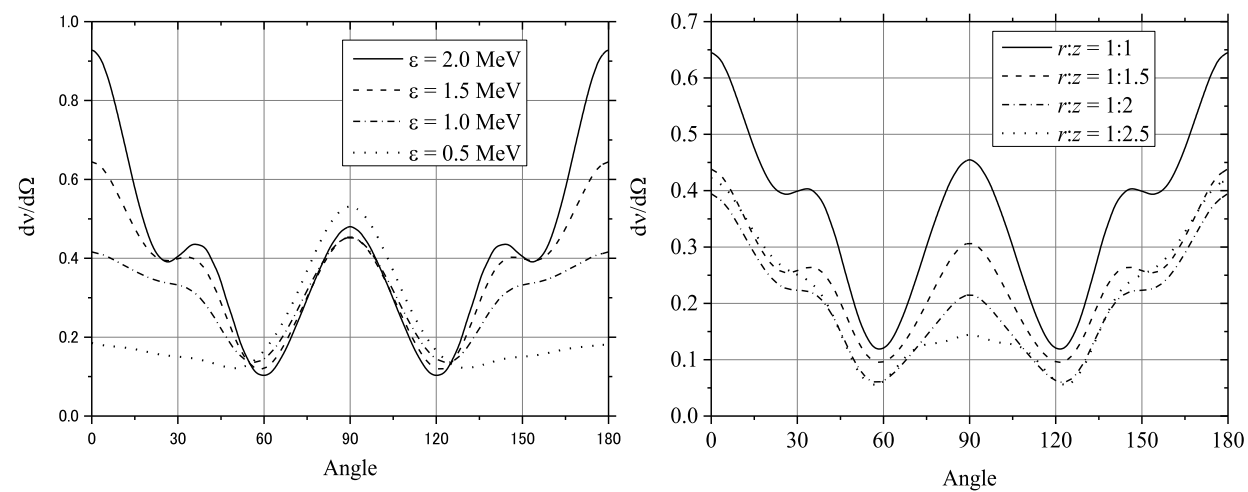

Figure 2. Angular distribution of scission neutron for asymmetric fission. Left panel: dependence on the motion of the fragments, Right panel: dependence on the deformation of the fragments.
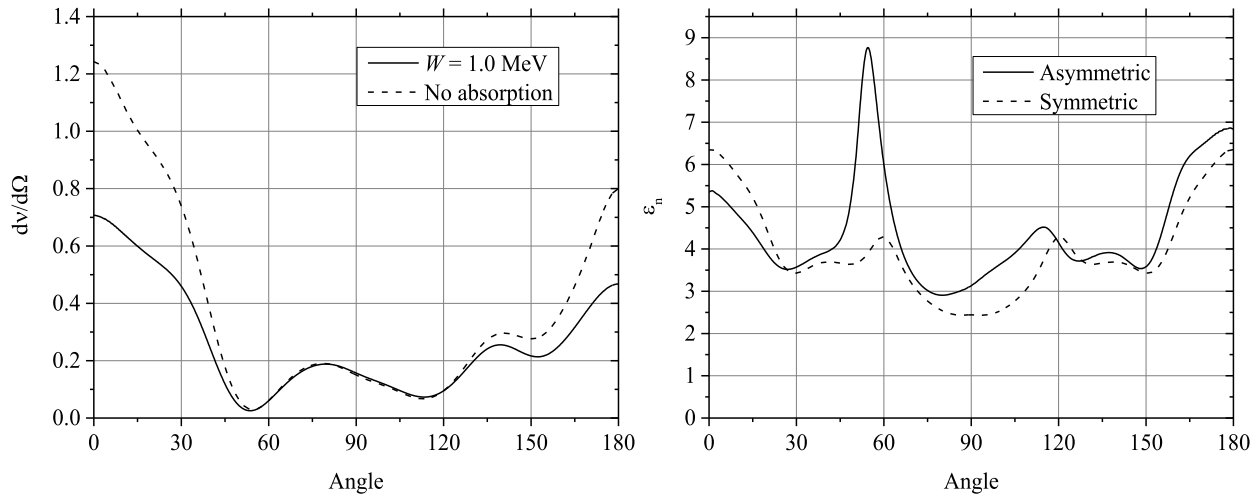

Figure 3. Left panel: comparison of the angular distribution of scission neutrons for the cases with and without absorption. Right panel: angular distribution of average energy of scission neutrons for symmetric and asymmetric fission.

calculated with $\left(W_{0}=-1 \mathrm{MeV}\right)$ and without $\left(W_{0}=0\right)$ the absorption. It is seen that the yields between 60 and 120 degrees do not change with the absorption, while the yields around 0 and 180 degrees are reduced significantly by the absorption.

The average energy of emitted neutron is calculated according to Eqs. (6)-(9). The right panel of Fig. 3 shows the angular distribution of the average energy for the symmetric and the asymmetric division. No precision kinetic energy is included and the absorption is set to $W_{0}=-1 \mathrm{MeV}$. It is seen that the average energy of neutron is high compared with the average kinetic energy of the initial wave function, $\langle\varepsilon\rangle=1.5 \mathrm{MeV}$ in this case. This is partly due to the motion of the fragments and is partly due to the finite duration of the calculation. Qualitatively speaking, slow component stays inside of the boundary for a long time, while the components with large kinetic energy arrive at the boundary faster. It is seen that the yields around 0 and 180 degrees have relatively high kinetic energy due to the attractioin of the fragments in both symmetric and asymmetric cases.

\subsection{Treatment of boundaries}

In order to avoid unrealistic reflections of the wave function at the border of the grid, there are essentially two methods; one is to prepare a large calculation grid and put a week absorbing potential 
outside of the sphere of radius $R$, on which we calculate the outgoing flux, the other is to use a smaller calculation grid and to use a proper artificial boundary condition to avoid the reflection. The former method has been employed in our calculation so far. One drawback of this method is that we need extra CPU time for the calculation outside of the physical region.

One example of the latter is a so-called "transparent boundary condition" (TBC) [6]. For the case of one-dimensional problem, it assumes that the wave function can be approximated by a single energy plane wave, i.e. $\psi(x) \propto e^{i k x}$. Because of the second differential in the kinetic energy term of the Hamiltonian, we need to extrapolate the wave function beyond the boundary. With TBC, the extrapolation is done as,

$$
\psi\left(x_{M}+\Delta x\right)=\frac{\psi\left(x_{M}\right)^{2}}{\psi\left(x_{M}-\Delta x\right)}
$$

where $x_{M}$ denotes the boundary point. When we denote $f(x)=\log (\psi(x))$, Eq. (11) corresponds to the linear approximation, $f(x+\Delta x)=2 f(x)-f(x-\Delta x)$. We now propose to use the secondorder approximation, $f(x+\Delta x)=3 f(x)-3 f(x-\Delta x)+f(x-2 \Delta x)$ to improve the accuracy of the extrapolation. We call this new method "second-order TBC" and applied it to a one-dimensional problem for free particle for which we know the exact solution. It was found that the error due to the extrapolation was much smaller compared with that with the original TBC. We also applied this method to the calculation of scission neutron. Though some fine tuning was necessary in the application, we obtained essentially the same accuracy as the one with the imaginary potential method but with less CPU time.

\section{Summary}

The effects of the scattering and re-absorption by the fission fragments on the angular distribution of scission particles have been investigated in the framework of the time-dependent Schrödinger equation. The effects of the fragments are taken account in terms of the nuclear optical potentials. We solve the time-dependent Schrödinger equation of the emitted neutron and the angular distribution is calculated by counting the outgoing flux. It has been demonstrated that the attractive nuclear potential enhances the yields around 0 and 180 degrees, while the absorptive potential diminishes them. In the case of asymmetric fission, the heavy fragment which is larger in size causes stronger effect to the angular distribution of scission neutrons. In order to elucidate the essential features of the effects of the fragments, we have examined the angular distribution by changing the initial wave function. We have also examined dependence on the position and the deformation of the fragments. The angular distribution of the average energy of emitted neutrons has been discussed. Finally, we have proposed a new boundary condition to minimize the reflection on artificial boundaries due to finiteness of the grid.

\section{References}

[1] N. Carjan, P. Talou, O. Serot, Nucl. Phys. A 792, 102 (2007)

[2] M. Rizea, N. Carjan, T. Wada, Physica Procedia 47, 27 (2013)

[3] T. Wada, T. Asano, Proc. 5th Int. Conf. on Fission and Properties of Neutron-Rich Nuclei (World Scientific, 2014), 409

[4] T. Wada, T. Asano, N. Carjan, Physica Procedia 47, 33 (2013)

[5] Y.L. Zhao et al., Phys. Rev. C 62, 14612 (2000)

[6] N. Carjan, M. Rizea, D. Storottman, Comp. Phys. Comm. 173, 41 (2005) 